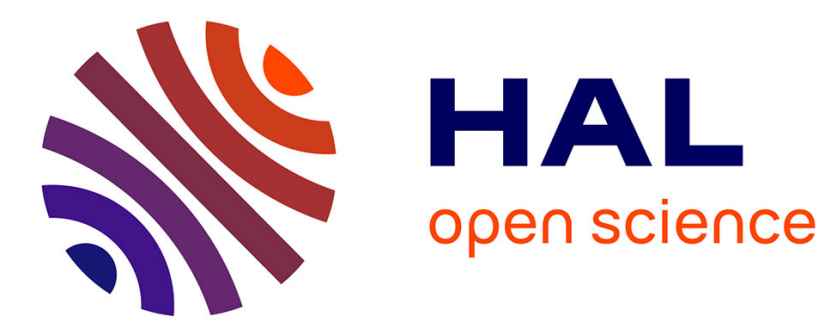

\title{
HUE-PRESERVING PERCEPTUAL CONTRAST ENHANCEMENT
}

Fabien Pierre, Jean-François Aujol, Aurélie Bugeau, Gabriele Steidl, Vinh-Thong Ta

\section{- To cite this version:}

Fabien Pierre, Jean-François Aujol, Aurélie Bugeau, Gabriele Steidl, Vinh-Thong Ta. HUEPRESERVING PERCEPTUAL CONTRAST ENHANCEMENT. Internationnal Conference on Image Processing (ICIP), Jun 2016, Phoenix, United States. hal-01316432

\section{HAL Id: hal-01316432 \\ https://hal.science/hal-01316432}

Submitted on 17 May 2016

HAL is a multi-disciplinary open access archive for the deposit and dissemination of scientific research documents, whether they are published or not. The documents may come from teaching and research institutions in France or abroad, or from public or private research centers.
L'archive ouverte pluridisciplinaire HAL, est destinée au dépôt et à la diffusion de documents scientifiques de niveau recherche, publiés ou non, émanant des établissements d'enseignement et de recherche français ou étrangers, des laboratoires publics ou privés. 


\title{
HUE-PRESERVING PERCEPTUAL CONTRAST ENHANCEMENT
}

\author{
Fabien Pierre $^{1,2}$, Jean-François Aujol ${ }^{1}$, Aurélie Bugeau ${ }^{2}$, Gabriele Steidl ${ }^{3}$, Vinh-Thong Ta ${ }^{2,4}$ \\ ${ }^{1}$ Univ. Bordeaux, IMB, CNRS, UMR 5251, INP, F-33400 Talence, France. \\ ${ }^{2}$ Univ. Bordeaux, LaBRI, CNRS, UMR 5800, F-33400 Talence, France. \\ ${ }^{3}$ Technische Universität Kaiserslautern, Fachbereich Mathematik, Postfach 3049, 67653 Kaiserslautern. \\ ${ }^{4}$ Bordeaux INP, LaBRI, UMR 5800, F-33405 Talence, France.
}

\begin{abstract}
This paper proposes a novel model for contrast enhancement of RGB images. The average local contrast measure is increased within a variational framework which preserves the hue of the original image by coupling the channels. The user is enabled to intuitively control the level of the contrast as well as the scale of the enhanced details. Moreover, our model avoids large modifications of the original image histogram and thereby preserves the global illumination of the scene. The minimizer of the proposed functional is computed by a hybrid primal-dual algorithm. Numerical experiments show the reliability of the proposed approach in comparison with state-of-the-art methods.
\end{abstract}

Index Terms - Contrast enhancement, variational methods, color correction.

\section{INTRODUCTION}

Contrast enhancement of color images that are over-lighted or under-lighted is needed to improve the quality of digital photography. Existing methods can be divided into three groups: spectral methods, histogram methods, and spatial methods.

Spectral methods rely either on wavelets or homomorphic filtering. In [1], homomorphic filtering is performed after a logarithmic transformation. Basically, the homomorphic filter is a high-pass filter which enforces the sharpness of the image. A similar approach has been developed with wavelets by Laine et al. [2].

Histogram Specification (HS) transforms the gray-scale input image to an output having a previously specified histogram. A special case of HS is Histogram Equalization, where the specified histogram is the uniform one. These methods have been well studied in the literature (see, e.g., $[3,4,5,6,7])$. In order to avoid problems in multimodal histograms, some approaches divide the histogram specification into sub-histogram specifications (see, e.g., [8]

This study has been carried out with financial support from the French State, managed by the French National Research Agency (ANR) in the frame of the Investments for the future Programme IdEx Bordeaux (ANR10-IDEX-03-02). J-F. Aujol is a member of Institut Universitaire de France. and the references therein). Some methods are adaptive because the enhancement is done locally, (see, e.g., [9] and the references therein). However, in these methods the influence of neighboring and far away pixels on the enhanced one is the same.

For color images, a two step algorithm consisting of a HS of the intensity image, followed by a fast hue and range preserving color adjustment was proposed by Nikolova et al. [10] (see [11] for available code).

The spatial methods tackle with the problem of most histogram approaches which change the values of pixels without considering spatial constraints. Most spatial methods take the Human Visual System (HSV) hypotheses into account. In particular, the perceptual work about Automatic Color Equalization (ACE) [12] has found wide acceptance. Bertalmio et al. [13] integrate the previous approach into a variational model. The method was generalized by Palma-Amestoy et al. [14] for a larger class of functionals. This last method was implemented in [15]. Provenzi et al. [16] propose to work in the wavelet domain in order to reduce the computation time. These approaches take into account the fact that two remote pixels with the same value can be perceived by a human as having different intensities. This phenomenon is called lateral inhibition (see, e.g., Checker shadow illusion of Adelson [17]).

Contributions. In this paper we propose a variational model increasing the average local contrast measure, preserving the hue and modelling the lateral inhibition. The control of the level of contrast can be tuned with an intuitive parameter, making it more adaptive. The proposed model enables the choice of the enhanced scale, which is significant for contrast enhancement. The proposed approach does not strongly modify the histogram of the original image. This enables the model to preserve the global lighting sensation. The proposed approach is composed of a single step which is reduced to the minimization of the functional.

Outline. In Section 2 we present a functional dedicated to the contrast enhancement of gray-scale images, we then recall the color model of [18] and we merge the two models within a variational framework. The functional is then min- 
imized by a hybrid primal-dual algorithm presented in Section 3. Finally, in Section 4, comparisons with state-of-theart methods demonstrate the efficiency of the proposed variational method.

\section{VARIATIONAL MODEL FOR COLOR IMAGE CONTRAST ENHANCEMENT}

In this section, we propose a new model for the enhancement of color images. We consider $M \times N$ digital images on the image grid $\Lambda:=\{1, \ldots, M\} \times\{1, \ldots, N\}$ with values in $[0,1]$. Let $\mathcal{B}:=[0,1]^{M \times N}$ a normalized range of images.

\subsection{One Channel Contrast Enhancement}

Our model is based on the enhancement of a contrast measure, the average local contrast measure introduced in [13]:

$$
\mathrm{C}(u):=\sum_{x \in \Lambda} \sum_{y \in \Lambda} w(x, y)|u(x)-u(y)|,
$$

where $w$ is a Gaussian kernel.

Now, our model for the enhancement of a given gray-scale image $u_{0} \in \mathcal{B}$ consists in minimizing the following functional

$$
F(u):=\left\|u-u_{0}\right\|_{2}^{2}+\lambda\left(\mathrm{C}(u)-c \mathrm{C}\left(u_{0}\right)\right)^{2}+\iota_{\mathcal{B}}(u) .
$$

Here $\iota_{\mathcal{B}}$ denotes the indicator function of $\mathcal{B}$ which is zero within the set $\mathcal{B}$ and $+\infty$ outside. It guarantees that the range of the image is preserved. The regularization parameter $\lambda>0$ manages the trade-off between the fidelity-data term and the contrast term. The constant $c>1$ implies that a minimizer of (2) increases the average local contrast measure. Intuitively, the contrast of the result tends to be $c$ times larger than the one of the input image. This can be interpreted as a multiplication by $c$ of the dynamic of the contours in the image. The choice of the parameter $c$ is intuitive and has a geometrical interpretation. It provides a control of the contrast level expected for the result. In practical cases, we use a derivable approximation of $\mathrm{C}$ denoted by $\tilde{\mathrm{C}}$ (see, e.g., [19]).

\subsection{Approximate Hue Preservation}

Model (2), is designed to enhance one-channel images. A simple generalization of our model to color images consists in treating each color channel separately. This method does not preserve the hue of the original image. In the following, we enhance color images through the contrast of the intensity channel.

In this paper, we focus on color image enhancement that respects the hue of the original image. Recall that the hue of a single pixel $u=(r, g, b)$ is given by $H(u):=0$ if $r=g=b$ and otherwise by

$$
H(u):= \begin{cases}\theta & \text { if } b \leq g \\ 360^{\circ}-\theta & \text { if } b>g\end{cases}
$$

where $\theta:=\arccos \frac{\frac{1}{2}((r-g)+(r-b))}{\left.\left((r-g)^{2}+(r-b)\right)(g-b)\right)^{\frac{1}{2}}}$. The definition of the hue considers white balanced images.

By the following proposition whose proof can be found, e.g., in [18], it follows immediately a characterization of colors having the same hue.

Proposition 1. The single pixels $\left(r_{0}, g_{0}, b_{0}\right) \in[0,1]^{3}$ and $(r, g, b) \in[0,1]^{3}$ have the same hue if and only if there exist $a, d \in \mathbb{R}$ such that $(r, g, b)=a\left(r_{0}, g_{0}, b_{0}\right)+d \mathbf{1}_{3}{ }^{\mathrm{T}}$, where $\mathbf{1}_{3}:=(1,1,1)^{\mathrm{T}}$.

Given a color image $u_{0}$ with intensity $I_{0}$, the authors of [18] propose a variational model to obtain an enhanced image $u$ with prescribed intensity $I$ that respects to some degree the hue of the original image. To this end, auxiliary variables

$$
x_{R}, x_{G}, x_{B} \in \mathbb{R}^{M \times N} \quad \text { and } \quad d \in \mathbb{R}^{M \times N}
$$

are introduced which are related to the desired (concatenated) image $u=(r, g, b)$ by

$$
r:=\frac{r_{0}}{I_{0}} \circ x_{R}+d, g:=\frac{g_{0}}{I_{0}} \circ x_{G}+d, b:=\frac{b_{0}}{I_{0}} \circ x_{B}+d .
$$

Here the quotients $A_{R}:=\frac{r_{0}}{I_{0}}, A_{G}:=\frac{g_{0}}{I_{0}}, A_{B}:=\frac{b_{0}}{I_{0}} \in$ $\mathbb{R}^{M \times N}$ are computed componentwise, and $\circ$ denotes the componentwise (Hadamard) product. If $I_{0}(x)=0$, we use $A_{R}=$ $A_{G}=A_{B}=1$. To shorten the notation, we set

$$
x_{R G B}:=\left(x_{R}, x_{B}, x_{G}\right) \in \mathbb{R}^{M \times N \times 3}
$$

and denote the assignment in (4) by

$$
u=(r, g, b)=\left(A x_{R G B}, d\right) .
$$

Clearly, if $x_{R} \approx x_{G} \approx x_{B}$, then $\frac{x_{\nu}}{I_{0}}, \nu \in\{R, G, B\}$ in (4) approximate the constant $a$ in Proposition 1 so that $u$ should respect the hue of $u_{0}$. We enforce the approximate hue preservation by penalizing

$$
\tilde{H}\left(x_{R G B}\right):=\left\|x_{R}-x_{G}\right\|_{2}^{2}+\left\|x_{R}-x_{B}\right\|_{2}^{2}+\left\|x_{G}-x_{B}\right\|_{2}^{2} .
$$

\subsection{New Variational Model for Color Images}

We extend Model (2) to the intensity of a color image parametrized by variable $x_{R G B}$ and $y$. To cope with noise while preserving the image edges we penalize the discrete total variation (TV) [20] of $u$. The total variation of $u$ in terms of $x_{R G B}$ and $d$ is given by

$$
T V\left(x_{R G B}, d\right)=\sum_{x \in \Lambda}\left(\sum_{\nu \in\{R, G, B\}}\left\|\nabla\left(A_{\nu} \circ x_{\nu}+d\right)(x)\right\|^{2}\right)^{\frac{1}{2}} .
$$

We penalize the hue drift by adding $\tilde{H}$ defined as in (6). 
The proposed unified model reads as follows:

$$
\begin{aligned}
\underset{x_{R G B}, d}{\arg \min } & \left\{\frac{\lambda}{2}\|d\|_{2}^{2}+\frac{\mu}{2} \tilde{H}\left(x_{R G B}\right)+\operatorname{TV}\left(x_{R G B}, d\right)\right. \\
& +\frac{\alpha}{2}\left(\tilde{\mathrm{C}}\left(I\left(x_{R G B}, d\right)\right)-c \tilde{\mathrm{C}}\left(I_{0}\right)\right)^{2} \\
& \left.+\frac{\beta}{2}\left\|I\left(x_{R G B}, d\right)-I_{0}\right\|_{2}^{2}+\iota_{\mathcal{B}^{3}}\left(A x_{R G B}, d\right) .\right\}
\end{aligned}
$$

If $\hat{x}_{R G B}, \hat{d}$ is a solution, then $\left(A \hat{x}_{R G B}, \hat{d}\right)$ is the desired image. The aim of this model is to enhance at the same time the intensity channel and to assign to each pixel a color whose hue is close to that of the original one. A high value of $\mu$ ensures that the hue of the new image is close to the original one. The scale of the contrast enhancement is controlled by the size of the kernel used for the computation of $\tilde{\mathrm{C}}$. The contrast level is tuned by the parameter $c$. The parameter $\lambda$ enforces the algorithm towards the multiplicative method of [10] so that it increases the colorfulness of the result.

\section{ALGORITHM}

Chambolle and Pock [21] propose an algorithm to compute a saddle-point of a problem of the form

$$
\min _{p} \max _{q}\langle K p, q\rangle+f(p)+g(p)-h^{*}(q),
$$

where $f$ has a Lipschitz gradient with constant $L_{f}$, and $g, h$ are proper, lower semi-continuous, convex functions with Fenchel dual $h^{*}$. Furthermore, $K$ is a bounded linear operator.

In order to apply the algorithm to our model (8), we reshape all images from $\mathbb{R}^{M \times N}$ columnwise into vectors of length $n=M N$. This is often described by the vec operation. Since the format becomes clear from the context we keep the notation $x$ instead of $\operatorname{vec}(x)$. In particular, we have $x_{R G B} \in \mathbb{R}^{3 n}$ and $d \in \mathbb{R}^{n}$ now. Moreover, after reshaping $A_{\nu}, \nu \in\{R, G, B\}$ into vectors of length $n$, we built the diagonal matrices $\boldsymbol{A}_{\nu}, \nu \in\{R, G, B\}$ out of these vectors. We can rewrite the total variation term for this setting by applying the vector adapted gradient operator $\nabla: \mathbb{R}^{n} \mapsto \mathbb{R}^{2 n}$ below

$\boldsymbol{\nabla}:=\left(\begin{array}{l}\mathfrak{I}_{N} \otimes D_{M} \\ D_{N} \otimes \mathfrak{I}_{M}\end{array}\right), \quad D_{M}:=\left(\begin{array}{cccc}-1 & 1 & & \\ & \ddots & \ddots & \\ & & -1 & 1 \\ & & & 0\end{array}\right) \in \mathbb{R}^{M, M}$,

where $\otimes$ denotes the Kronecker product and $\mathfrak{I}_{N}$ is the $N \times N$ identity matrix. Setting

$$
\begin{aligned}
f\left(x_{R G B}, d\right) & :=\frac{\lambda}{2}\|d\|_{2}^{2}+\frac{\beta}{2}\left\|I\left(x_{R G B}, d\right)-I_{0}\right\|_{2}^{2} \\
+\frac{\alpha}{2}\left(\tilde{\mathrm{C}}\left(I\left(x_{R G B}, d\right)\right)\right. & \left.-c \tilde{\mathrm{C}}\left(I_{0}\right)\right)^{2}+\frac{\mu}{2} \tilde{H}\left(x_{R G B}\right), \\
g\left(x_{R G B}, d\right) & :=0 \\
h(v, u) & :=\|v\|_{2,1}+\iota_{[0,1]^{3 n}}(u)
\end{aligned}
$$

and

$$
K:=\left(\begin{array}{c|c}
\left(\mathfrak{I}_{3} \otimes \boldsymbol{\nabla}\right) \boldsymbol{A} & \mathbf{1}_{3} \otimes \boldsymbol{\nabla} \\
\hline \boldsymbol{A} & \mathbf{1}_{3} \otimes \mathfrak{I}_{n}
\end{array}\right), \quad \boldsymbol{A}:=\operatorname{diag}\left(A_{R}, A_{G}, A_{B}\right) .
$$

Functional (8) is minimized by Algorithm 1.

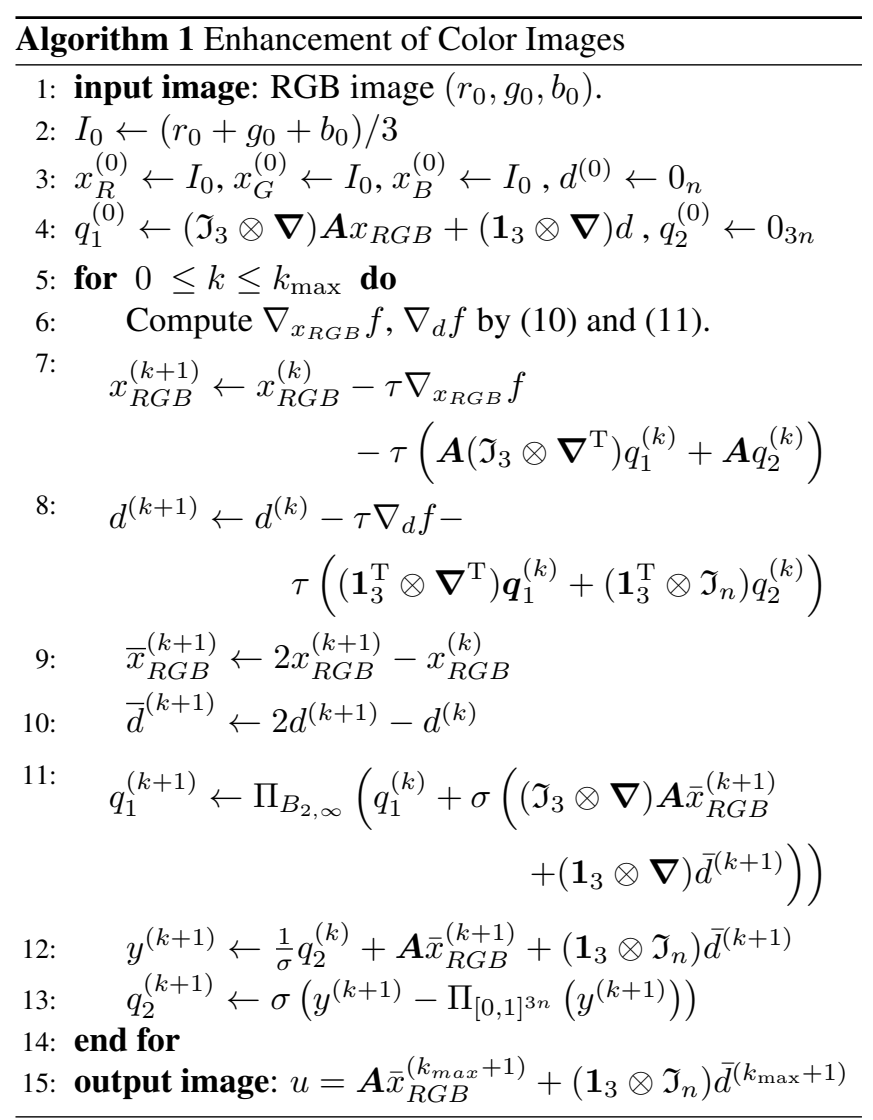

$\Pi_{B_{2, \infty}}$ (resp. $\left.\Pi_{[0,1]^{3 n}}\right)$ is the projection onto the $L^{2}$ unit ball (resp. onto the pixelwise cube $[0,1]^{3 n}$ ).

The most expensive computation is the one of $\tilde{\mathrm{C}}$ and $\nabla \tilde{\mathrm{C}}$ embedded in the computation of $\nabla f$. This computation is accelerated in the same way as in [13].

Computation of $\nabla f=\nabla_{x_{R G B}} f, \nabla_{d} f$. The derivative of $f$ with respect to $x_{\nu}, \nu=R, G, B$ is given by

$$
\begin{aligned}
& \frac{\partial f}{\partial x_{\nu}}=\mu\left(2 x_{\nu}-x_{\hat{\nu}}-x_{\tilde{\nu}}\right)+\frac{\beta \nu_{0}}{3 I_{0}}\left(I\left(x_{R G B}, d\right)-I_{0}\right)+ \\
& \frac{\alpha \nu_{0}}{3 I_{0}} \nabla \tilde{\mathrm{C}}\left(I\left(x_{R G B}, d\right)\right)\left(\tilde{\mathrm{C}}\left(I\left(x_{R G B}, d\right)\right)-c \tilde{\mathrm{C}}\left(I_{0}\right)\right),
\end{aligned}
$$

where $\tilde{\nu}, \hat{\nu} \in\{R, G, B\}$. The derivative of $f$ with respect to $d$ reads

$$
\begin{array}{r}
\frac{\partial f}{\partial d}=\lambda d+\alpha \nabla \tilde{\mathrm{C}}\left(I\left(x_{R G B}, d\right)\right)\left(\tilde{\mathrm{C}}\left(I\left(x_{R G B}, d\right)\right)-c \tilde{\mathrm{C}}\left(I_{0}\right)\right) \\
+\beta\left(I\left(x_{R G B}, d\right)-I_{0}\right) .
\end{array}
$$



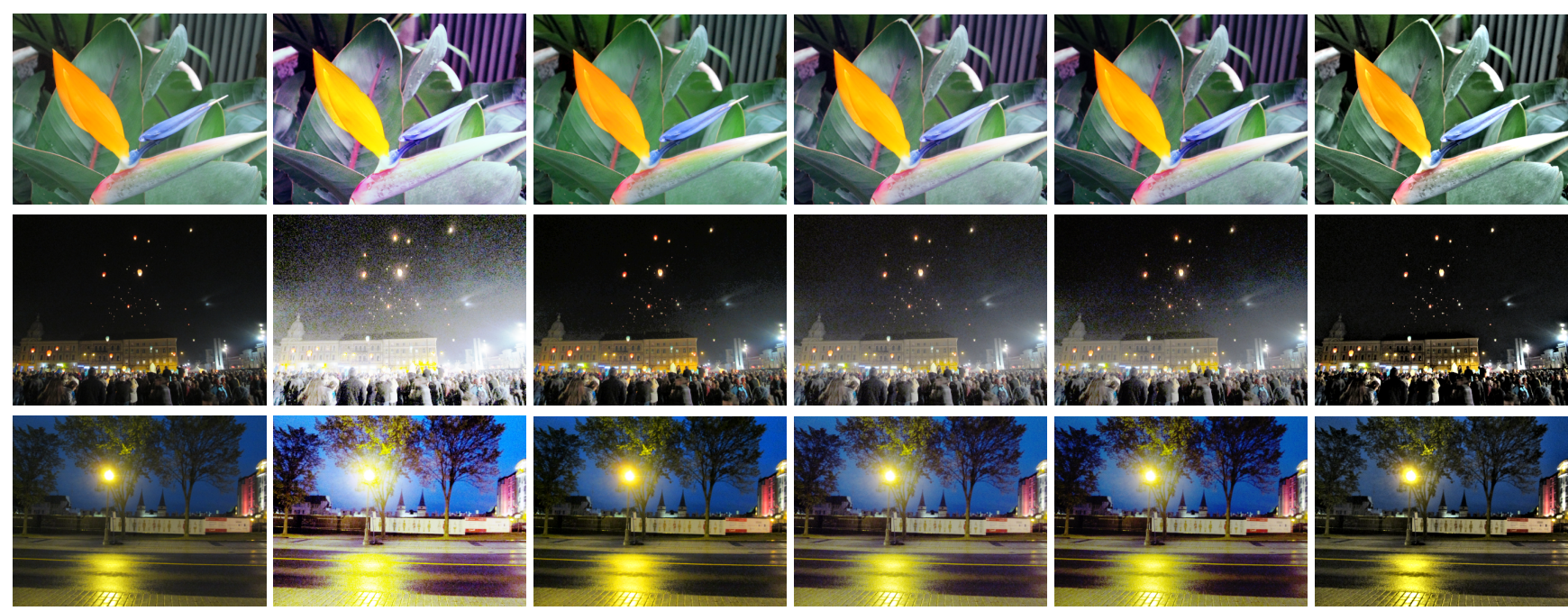

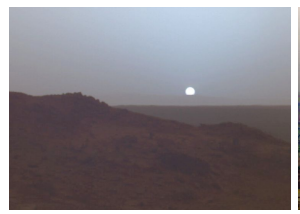

Original

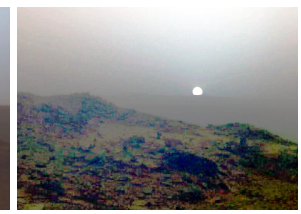

Gimp

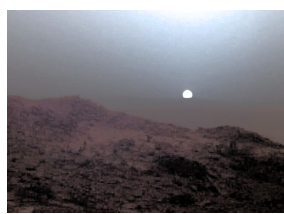

[10]

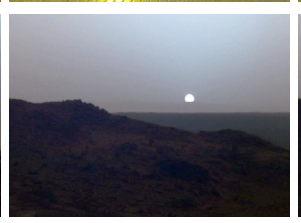

[13]

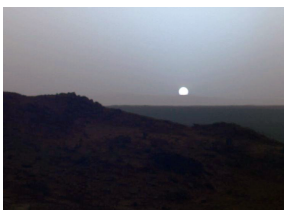

[15]

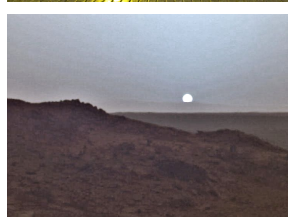

Ours

Fig. 1. Comparison of our results with those obtained by the algorithms of the Gimp histogram equalization, of Nikolova et al. [10], of Bertalmio et al. [13], of Ferradans et al. [15]. The proposed algorithm produces reliable results in all these cases.

\section{NUMERICAL RESULTS}

In this section, Algorithm 1 is applied to color images and the results are compared to some state-of-the-art methods. The algorithm is implemented in MATLAB.

The following parameters have been experimentally chosen: $\lambda=100, \mu=1, \alpha=100, \beta=500, \sigma=5.10^{-5}$, $\tau=5.10^{-9}$ and $10^{3}$ iterations. The polynomial approximation of $\mathrm{C}$ is done with a primitive of the Bernstein approximation of the sign function, with degree 9 (see, e.g., [19]). The kernel $w$ of $\mathrm{C}$ is a Gaussian one, with a size related to the image one. In experiments it is chosen equal to $\min (M, N) / 20$. This size manages the scale of the contrast enhancement.

In Figure 1, we compare Algorithm 1 to the equalization implementation of Gimp, the algorithm of Nikolova et al. [10, 22] with implementation [11], the method of Bertalmio et al. [13] and the one of Ferradans et al. [15] with entropic Michelson function. For the method of [13], we use the implementation [15]. For the comparison approaches, we used the default parameters of the papers. The implementation of Gimp consists in a histogram equalization of the intensity channel. This method is not able to preserve the hue, because the transformation from the HSV color-space to RGB can change the hue (see, e.g., [23]).

On the Flower image, the approaches of $[13,15]$ as well as the one of the Gimp changes the hue, thus, the background becomes purple. The proposed approach, as well as the one of [10] preserves the hue and the colorfulness.

In the $2^{\text {nd }}$ line, the original image has been taken with a standard customer camera during the night and is very noisy. Contrary to other approaches, the one proposed in this paper is able to cope with noisy pixels due to the TV regularization, and the noise is not amplified.

The $3^{\text {rd }}$ line shows a night image. In our result, the sky is darker than the results with methods of $[13,15]$, that preserves the sensation of a photography taken during the night.

In the Mars landscape the methods of $[13,15]$ produce images with the bottom of the image which is too dark, in comparison with the original image. The global illumination and appearance of the image is preserved with our model.

Additional results are available in [24] or at: http://www.labri.fr/perso/fpierre/eh/enhancement.html

\section{SUMMARY AND CONCLUSIONS}

We introduced a novel variational model for the enhancement of color images. Our enhancement method works directly on the RGB image without decoupling of the color channels or enhancing the intensity in a previous step. We demonstrated that our model is able to enhance contrast on images where state-of-the-art methods fail. The automatic tuning of contrast level and scale will be studied in a future work. 


\section{REFERENCES}

[1] Rafael C Gonzalez and Richard E Woods, "Digital image processing 3rd edition," 2007.

[2] Andrew Laine, Jian Fan, and Wuhai Yang, "Wavelets for contrast enhancement of digital mammography," IEEE Engineering in Medicine and Biology Magazine, vol. 14, no. 5, pp. 536-550, 1995.

[3] Guillermo Sapiro and Vicent Caselles, "Histogram modification via differential equations," Journal of Differential Equations, vol. 135, no. 2, pp. 238-268, 1997.

[4] Chi-Chia Sun, Shanq-Jang Ruan, Mon-Chau Shie, and Tun-Wen Pai, "Dynamic contrast enhancement based on histogram specification," IEEE Transactions on Consumer Electronics, vol. 51, no. 4, pp. 1300-1305, 2005.

[5] Chao Wang and Zhongfu Ye, "Brightness preserving histogram equalization with maximum entropy: a variational perspective," IEEE Transactions on Consumer Electronics, vol. 51, no. 4, pp. 1326-1334, 2005.

[6] Max Mignotte, "An energy-based model for the image edge-histogram specification problem," IEEE Transactions on Image Processing, vol. 21, no. 1, pp. 379-386, 2012.

[7] Tarik Arici, Salih Dikbas, and Yucel Altunbasak, "A histogram modification framework and its application for image contrast enhancement," IEEE Transactions on Image Processing, vol. 18, no. 9, pp. 1921-1935, 2009.

[8] Manpreet Kaur, Jasdeep Kaur, and Jappreet Kaur, "Survey of contrast enhancement techniques based on histogram equalization," International Journal of $\mathrm{Ad}$ vanced Computer Science and Applications, vol. 2, no. 7, pp. 137-141, 2011.

[9] Raman Maini and Himanshu Aggarwal, "A comprehensive review of image enhancement techniques," Journal of Computing, vol. 2, no. 3, pp. 919-940, 2010.

[10] Mila Nikolova and Gabriele Steidl, "Fast hue and range preserving histogram specification: Theory and new algorithms for color image enhancement," IEEE Transactions on Image Processing, vol. 23, no. 9, pp. 40874100, 2014.

[11] Sören Häuser, Mila Nikolova, and Gabriele Steidl, "Hue and range preserving RGB image enhancement (RGBHP-ENHANCE)," Preprint, 2015, Documentation for Matlab toolbox.

[12] Alessandro Rizzi, Carlo Gatta, and Daniele Marini, "A new algorithm for unsupervised global and local color correction," Pattern Recognition Letters, vol. 24, no. 11, pp. 1663-1677, 2003.
[13] Marcelo Bertalmío, Vicent Caselles, Edoardo Provenzi, and Alessandro Rizzi, "Perceptual color correction through variational techniques," IEEE Transactions on Image Processing, vol. 16, no. 4, pp. 1058-1072, 2007.

[14] Rodrigo Palma-Amestoy, Edoardo Provenzi, Marcelo Bertalmío, and Vincent Caselles, "A perceptually inspired variational framework for color enhancement," IEEE Transactions on Pattern Analysis and Machine Intelligence, vol. 31, no. 3, pp. 458-474, 2009.

[15] Sira Ferradans, Rodrigo Palma-Amestoy, and Edoardo Provenzi, "An algorithmic analysis of variational models for perceptual local contrast enhancement," Image Processing On Line, vol. 5, pp. 219-233, 2015.

[16] Edoardo Provenzi and Vicent Caselles, "A wavelet perspective on variational perceptually-inspired color enhancement," International Journal of Computer Vision, vol. 106, no. 2, pp. 153-171, 2014.

[17] Edward H Adelson, "Checkershadow illusion," Available at http://persci.mit.edu/gallery/checkershadow, vol. 2, no. 1, 1995.

[18] Jan Henrik Fitschen, Mila Nikolova, Fabien Pierre, and Gabriele Steidl, "A variational model for color assignment," in Scale Space and Variational Methods in Computer Vision, 2015, pp. 437-448.

[19] Fabien Pierre and Pauline Migerditichan, "Débrumage variationnel," in XXVème colloque GRETSI, 2015, pp. $1-4$.

[20] Leonid I Rudin, Stanley Osher, and Emad Fatemi, "Nonlinear total variation based noise removal algorithms," Physica D: Nonlinear Phenomena, vol. 60, no. 1, pp. 259-268, 1992.

[21] Antonin Chambolle and Thomas Pock, "On the ergodic convergence rates of a first-order primal-dual algorithm," in Mathematical Programming, 2015.

[22] Mila Nikolova and Gabriele Steidl, "Fast ordering algorithm for exact histogram specification," IEEE Transactions on Image Processing, vol. 23, no. 12, pp. 52745283, 2014.

[23] Fabien Pierre, Jean-François Aujol, Aurélie Bugeau, and Vinh-Thong Ta, "Luminance-hue specification in the RGB space," in Scale Space and Variational Methods in Computer Vision, 2015, pp. 413-424.

[24] Fabien Pierre, Jean-François Aujol, Aurélie Bugeau, Gabriele Steidl, and Vinh-Thong Ta, "Variational Contrast Enhancement of RGB Images," preprint available at https://hal.archives-ouvertes.fr/hal01237038v4/document, Dec. 2015. 